\title{
Die Verwandtschaft von Philosophie und Religion Erinnerung an ein verdrängtes Sachproblem
}

\author{
Prof. Dr. Axel Hutter, Ludwig-Maximilians-Universität München, \\ Geschwister-Scholl-Platz 1, 80539 München, axel.hutter@lmu.de
}

$I$.

Das Sachproblem, das in dem folgenden Gedankengang vergegenwärtigt, nicht gelöst werden soll, ist dem heutigen Bewußtsein fremd geworden - und diese Fremdheit bildet ein wesentliches Moment der Problematik, an die hier in kritischer Absicht erinnert werden soll. Die Fremdheit für das Denken der Gegenwart, der spontane Eindruck, die anvisierte Fragestellung sei völlig unzeitgemäß, vom stetigen Fortschritt der menschlichen Einsicht überholt und glücklich in das Verzeichnis der überwundenen Verirrungen verbannt worden, - diese Irritation soll deshalb gar nicht verschwiegen oder auch nur verkleinert werden. Ganz im Gegenteil: Das Unzeitgemäße der Fragestellung muß zu Beginn ausdrücklich herausgestellt werden, da nur sie den konkreten Ausgangspunkt für einen Gedankengang bilden kann, der durch eine korrektive Erinnerung darauf abzielt, die dogmatische Enge des Gegenwartsbewußtseins kenntlich zu machen, die vor allem die zeitliche Enge eines vorschnellen und gewaltsamen Vergessens ist.

Ein wichtiger Orientierungspunkt für die anvisierte Erinnerung bildet das Denken Hegels, bei dem sich die folgende programmatische Bestimmung findet: "Der Gegenstand der Religion wie der Philosophie ist die ewige Wahrheit, Gott und nichts als Gott und die Explikation Gottes. Die Philosophie expliziert nur sich, indem sie die Religion expliziert, und indem sie sich expliziert, expliziert sie die Religion. Der denkende Geist ist es, der diesen Gegenstand, die Wahrheit, durchdringt [...] So fallen Religion und Philosophie in eins zusammen. Die Philosophie ist in der Tat selbst Gottesdienst, wie die Religion «. ${ }^{1} \mathrm{Da}$ diese Sätze, die mit großem Nachdruck eine grundlegende systematische Verwandtschaft von Philosophie und Religion artikulieren, dem heutigen Denken radikal befremdend, ja skandalös erscheinen müssen, wird kaum jemand in Abrede stellen wollen; viel weniger deutlich ist freilich das Sachproblem, das sich in ihnen andeutet, da es zunächst einmal von der Fremdheit völlig überlagert und verdeckt wird.

Immerhin läßt sich aus dem angeführten Zitat ein erster Hinweis entnehmen, sobald die Aufmerksamkeit auf die von Hegel verwendete Begriffskonstellation gelenkt wird. Die in Frage stehende Verwandtschaft ist

1 Georg Wilhelm Friedrich Hegel, Vorlesungen über die Philosophie der Religion, hg. v. Walter JAESCHKE, Bd. 1, Hamburg 1993, 63f. 
nämlich nicht etwa so zu verstehen, daß Philosophie und Religion schlicht identisch wären, sondern so, daß sie sich beide wesentlich auf denselben Gegenstand beziehen und durch diesen Bezug allererst zu dem werden, was sie jeweils spezifisch sind. ${ }^{2}$ Dieser gemeinsame Gegenstand kann aber mit verschiedenen Ausdrücken bezeichnet werden - und jede Bezeichnung wird in der Regel eine eher philosophische oder religiöse Färbung aufweisen. So verweist »Wahrheit « eher in einen philosophischen, "Gott « hingegen in einen religiösen Kontext, während der Begriff einer »ewigen Wahrheit« als Vermittlung zwischen beiden Kontexten verstanden werden kann. In jedem Fall ist es aber der »denkende Geist ", der »diesen Gegenstand, die Wahrheit, durchdringt «, so daß die systematische Verwandtschaft von Philosophie und Religion des Näheren als epistemische Verwandtschaft zu verstehen ist, die sich allein im Medium des Denkens und des Geistes genauer explizieren läßt.

Mag damit Hegels Doppelthese, die Philosophie expliziere nur sich, indem sie die Religion expliziere, und indem sie sich selbst expliziere, expliziere sie die Religion, etwas deutlicher geworden sein, so muß freilich weiterhin anerkannt werden, daß sich damit der Eindruck des Befremdlichen der These keineswegs verflüchtigt, womöglich sogar noch verstärkt hat. Schließlich war sich bereits Hegel völlig darüber im Klaren, daß eine Philosophie, die »versucht, mit der Erkenntnis Gottes sich einzulassen ", damit rechnen muß, »daß man gar nicht einmal acht darauf hat «, weil »ein solcher Gedanke ein längst widerlegter Irrtum sei. « ${ }^{3}$ Die Haltung des Zeitgeistes gegenüber dem Gedanken einer systematischen Verwandtschaft von Philosophie und Religion ist seitdem sicherlich nicht günstiger geworden, so daß der hier verfolgte Gedankengang gute Gründe hat, die innere Struktur dieser allgemein verbreiteten Ablehnung etwas ausführlicher zu thematisieren. Es wird sich zeigen, daß dieser »Umweg « sich der Sache nach nicht von Hegel entfernt, sondern geeignet ist, sehr genau in das Sachproblem einzuführen, das seine Philosophie in Atem hält.

II.

Die implizite Voraussetzung der heute allgemein verbreiteten Überzeugung, der Gedanke einer Verwandtschaft von Philosophie und Religion sei »ein längst widerlegter Irrtum ", läßt sich in einem ersten Anlauf als Drei-

2 Die durch den gemeinsamen Gegenstand begründete Wesensverwandtschaft schließt also eine Differenz zwischen Philosophie und Religion nicht aus, sondern fordert sie, weil sonst nicht von Verwandtschaft, sondern von Identität gesprochen werden müßte. Freilich rührt die Differenz nicht von einem unterschiedlichen Gegenstand her, sondern von einer unterschiedlichen Art und Weise, sich auf ein und denselben Gegenstand zu beziehen.

3 Hegel, Religion (s.o., Anm. 1), 6 (vgl. Georg Wilhelm Friedrich Hegel, Gesammelte Werke, hg. v. Walter JAesCHKe, Bd. 17, Hamburg 1987, 8). 
Stadien-Gesetz (Comte) der menschlichen Wissensentwicklung ausformulieren, demzufolge die Religion als erstes Entwicklungsstadium zu verstehen und somit der naiven Kindheit zu vergleichen ist. Auf die Religion folgt die Metaphysik (wie die Kindheit von einer in sich zerrissenen Pubertät abgelöst wird), in der sich der erwachende Verstand noch nicht konsequent durchsetzen kann, weil das menschliche Bewußtsein weiterhin in kindlichen Träumen und Wünschen befangen bleibt. Erst das dritte Stadium der positiven Wissenschaften, die sich in erwachsener Nüchternheit und Strenge ausschließlich an der empirisch beobachtbaren Realität ausrichten, wird das menschliche Wissen von seinen anfänglichen Verwirrungen auf Dauer befreien, so daß nun endlich die feste Grundlage für einen stetigen, ungefährdeten Wissensfortschritt gelegt ist.

Gegen ein solches Entwicklungsschema, in dem sich das heutige Bewußtsein recht hochmütig mit dem bislang erreichten Höhepunkt identifiziert, kritische Einwände aufzubieten, ist von vornherein ein mißliches Unterfangen, da es sich in jedem Fall - ganz unabhängig von der möglichen Triftigkeit der Argumente - dem Verdacht aussetzt, bloß auf ein ohnmächtiges "Zurück zu besseren alten Tagen « hinauszulaufen. Deshalb soll hier die fortschrittsgläubige Selbstimmunisierung nicht von außen, sondern von innen her, das heißt im Ausgang von ihrem eigenen Selbstverständnis in eine selbstkritische Reflexionsbewegung versetzt werden. Das 20. Jahrhundert hat nämlich in einem bestürzenden Ausmaß Erfahrungen gemacht, die den naiven Fortschrittsglauben des vorangegangenen Jahrhunderts als selbstgefällige Illusion entlarven. Konsequenterweise beginnen deshalb gerade die besonders avancierten Vertreter einer streng wissenschaftlichen Orientierung damit, den Monopolanspruch der positiven Wissenschaften in Frage zu stellen. ${ }^{4}$ Für den hier verfolgten Gedankengang haben daher insbesondere jene pointiert formulierten Reflexionen zur Grenze der positiven Wissenschaften Gewicht, die der Siegeszug des wissenschaftlichen Positivismus bei denjenigen hervorruft, die selbst durchaus zu seinen prominenten Vertretern in den ersten Jahrzehnten des 20. Jahrhunderts zu zählen sind.

In diesem Sinne ist es bemerkenswert, daß ein Wissenschaftstheoretiker wie Carnap, der zu Beginn der dreißiger Jahre in seinem Aufsatz »Überwindung der Metaphysik durch logische Analyse der Sprache " dem Drei-Stadien-Gesetz noch einmal Referenz erweist, wenig später in einem kleinen Aufsatz festhält: »Wenn ich mir klar werden will, ob ich den vor mir liegenden Apfel essen soll oder nicht, so ist das eine Sache des Entschlußes, der praktischen Entscheidung, nicht der theoretischen. [...] Weder mein eigenes Denken noch alle Lehren der Wissenschaft sind imstande, jene scheinbare Frage zu beantworten; nicht als gäbe es hier eine Grenze des menschlichen

${ }^{4}$ Es gehört zur geistigen Physiognomie des 20. Jahrhunderts, daß der positivistische Wissenschafts- und Fortschrittsglaube zunehmend populär und zu einer neuen Volksreligion wird, während gleichzeitig die eigentlichen Protagonisten und »Insider " der modernen Wissenschaftskultur diesem Glauben mit Skepsis, ja zuweilen geradezu mit Verachtung begegnen. 
Verstandes, sondern einfach, weil gar keine Frage vorliegt. Theoretisch durch alltägliches oder wissenschaftliches Wissen - kann nur gesagt werden: swenn du den Apfel ißt, so wird dein Hunger verschwinden< (oder: sso wirst du dich vergiften`, sso wirst du ins Gefängnis kommen theoretischen Angaben über die zu erwartenden Folgen können gewiß für mich sehr wichtig sein; aber durch sie kann mir der Entschluß nicht abgenommen werden. Es ist Sache des praktischen Entschlußes, ob ich mich sättigen oder hungrig bleiben will; ob ich mich vergiften oder gesund bleiben will. $\ll^{5}$

Bemerkenswert ist an dieser Überlegung weniger, daß in ihr die Reichweite der Wissenschaft eingeschränkt wird, sondern wie diese Einschränkung erfolgt. Dort, wo die Wissenschaft für Carnap überhaupt etwas erkennen kann, ist ihre Erkenntnis grundsätzlich absolut: Es gibt keine »Grenze des menschlichen Verstandes «. Allerdings gibt es Problembereiche, in denen die Wissenschaft nichts erkennen kann, weil es dort - Carnap zufolge - nichts zu erkennen gibt (so daß das Nichterkennen nicht einer "Grenze " des Erkennens geschuldet, sondern schlicht eine »Eigenschaft « dieser Problembereiche ist). Es wäre also völlig unvernünftig, die Frage "Was soll ich tun? « mit Kant als Vernunftfrage zu verstehen, weil hier in Wahrheit "gar keine Frage vorliegt", so daß das Ausbleiben einer begründeten Antwort auch nicht als Mangel zu verstehen ist. ${ }^{6}$

Eine frühere und zugleich radikalere Fassung dieser Überlegung - die Carnap vermutlich zum Vorbild diente - stellt Wittgensteins "Logisch-philosophische Abhandlung « dar, in der sich gegen Ende der Satz findet: »Wir fühlen, daß selbst, wenn alle möglichen wissenschaftlichen Fragen beantwortet sind, unsere Lebensprobleme noch gar nicht berührt sind «(6.52). ${ }^{7}$ Ganz entsprechend schreibt Wittgenstein im Vorwort der Abhandlung: Die »Wahrheit der hier mitgeteilten Gedanken « scheint mir »unantastbar und definitiv. Ich bin also der Meinung, die Probleme im Wesentlichen endgültig gelöst zu haben. Und wenn ich mich hierin nicht irre, so besteht nun der Wert dieser Arbeit zweitens darin, daß sie zeigt, wie wenig damit getan ist, daß diese Probleme gelöst sind. ${ }^{8}$ Die eigentümliche Mischung aus Selbstsicherheit und Resignation, mit der hier Wittgenstein im Schatten des Ersten Weltkriegs über die Reichweite der wissenschaftlichen Fragen und Antworten nachdenkt, markiert eine Zäsur; die positiven Wissenschaften

5 Rudolf CARnap, Theoretische Fragen und praktische Entscheidungen, in: Natur und Geist 2 (1934), 257-60, hier 258.

6 Was bei der beliebten Trennung von Sein und Sollen kaum eigens reflektiert wird, ist die naheliegende Frage, welchen epistemischen Status diese stets in einem überraschend apodiktischen Ton vorgetragene Trennung von Tatsachen und Werten eigentlich hat: Ist diese These selbst eine (wissenschaftliche) Tatsache oder eine (lebenspraktische) Wertung? In beiden Fällen wäre aber die behauptete Trennung durch ihre Behauptung bereits aufgehoben.

7 Ludwig Wittgenstein, Logisch-philosophische Abhandlung. Werkausgabe, Bd. 1, Frankfurt a. M. 1995, 85 .

8 AaO., 10. 
behaupten zwar weiterhin ihr Selbstbewußtsein, für bestimmte Fragen in einer absoluten Weise zuständig zu sein, doch paart sich das Selbstbewußtsein nun mit der Einsicht, daß es andere Fragen gibt, für die sie in ebenso absoluter Weise unzuständig sind - und die letzteren Fragen sind gerade die wichtigen und entscheidenden.

Wittgensteins »Abhandlung " geht buchstäblich aufs Ganze, um ihre These von der strikten Trennung zwischen den Tatsachen der Wissenschaft und den Entscheidungsproblemen des Lebens (Wertungen) zu begründen: »Der Sinn der Welt muß außerhalb ihrer liegen. In der Welt ist alles wie es ist und geschieht alles wie es geschieht; es gibt in ihr keinen Wert - und wenn es ihn gäbe, so hätte er keinen Wert « (6.41). Daraus folgt dann notwendig die Schlußfolgerung, die sich später auch Carnap zu eigen machen wird: »Darum kann es auch keine Sätze der Ethik geben. Sätze können nichts Höheres ausdrücken « (6.42). Sind aber dergestalt erst einmal das Gefühl, die Lebensprobleme und die »Werte « zu absoluten Gegeninstanzen des in irgendeinem Sinne Begründbaren und Mitteilbaren avanciert, dann ist es nur noch ein kleiner Schritt zu dem berühmten Schlußdiktum, in dem sich Wittgensteins Abhandlung zu einer überaus prägnanten Abbreviatur verdichtet: "Wovon man nicht sprechen kann, darüber muß man schweigen.« (7)

Die philosophische Bedeutung der »Abhandlung « liegt aber nicht nur in ihrer rückhaltlosen Konsequenz, die sie am Ende ins Schweigen führt, sondern vielleicht mehr noch in der nicht ganz vermiedenen Inkonsequenz, über das, worüber man eigentlich schweigen muß, doch noch etwas zu sagen. So läßt sich Wittgenstein nicht nur zu dem Satz bewegen: »Es gibt allerdings Unaussprechliches. Dies zeigt sich, es ist das Mystische« (6.522); er setzt sogar noch hinzu: »Die Anschauung der Welt sub specie aeterni ist ihre Anschauung als - begrenztes - Ganzes. Das Gefühl der Welt als begrenztes Ganzes ist das mystische. " (6.45) Das am Ende geforderte Schweigen gibt sich an diesen Stellen als eine Form des »beredten "Schweigens zu erkennen; und das, was im Schweigen beredt verschwiegen wird, ist ein allerdings noch sehr unbestimmter - religiöser Kontext, der mit dem vagen Begriff des "Mystischen " evoziert wird und der des Weiteren als Ewigkeit bezeichnet wird, von der her die Welt in ihrer Begrenztheit und Endlichkeit »angeschaut « wird.

\section{III.}

Wittgensteins Dichotomie zwischen dem, wovon man sprechen kann, und dem, was stumm sich zeigt, besitzt eine eigentümliche Suggestionskraft, die ihre Macht freilich nicht aus ihrer sachlichen Angemessenheit bezieht, sondern daraus, daß sie bequemerweise den Umstand verschleiert, daß die »höheren « Werte in ihrer Pluralität untereinander im Konflikt stehen können, so daß das »Höhere« nicht die »mystische« Beruhigung aller 
Fragen, sondern auch und gerade die Quelle der wirklich beunruhigenden Fragen darstellt. ${ }^{9}$

Daß diese Suggestion zwar bequem, der Sache aber unangemessen ist, macht Max Weber genau zeitgleich zu Wittgensteins "Abhandlung « deutlich: "Die Unmöglichkeit 'wissenschaftlicher Vertretung von praktischen Stellungnahmen - außer im Falle der Erörterung der Mittel für einen als fest gegeben vorausgesetzten Zweck - folgt aus weit tiefer liegenden Gründen. Sie ist prinzipiell deshalb sinnlos, weil die verschiedenen Wertordnungen der Welt in unlöslichem Kampf untereinander stehen. " ${ }^{10}$ Gegen die ihm wohl vertraute moderne Tendenz, die Wertkonflikte zu verdecken und zu neutralisieren, beharrt Weber darauf, der Wahrheit ins Gesicht zu sehen: "Jede empirische Betrachtung dieser Sachverhalte « muß, so Weber, "zur Anerkennung des absoluten Polytheismus [...] führen. Eine nicht empirische, sondern sinndeutende Betrachtung: eine echte Wertphilosophie also, würde ferner, darüber hinausgehend, nicht verkennen dürfen, daß ein noch so wohlgeordnetes Begriffsschema der 'Werter gerade dem entscheidendsten Punkt des Tatbestandes nicht gerecht würde. Es handelt sich nämlich zwischen den Werten letztlich überall und immer wieder nicht nur um Alternativen, sondern um unüberbrückbar tödlichen Kampf, so wie zwischen >Gott< und >Teufek. Zwischen diesen gibt es keine Relativierungen und Kompromisse. Wohlgemerkt: dem Sinn nach nicht. Denn es gibt sie, wie jedermann im Leben erfährt, der Tatsache und folglich dem äußeren Schein nach, und zwar auf Schritt und Tritt. In fast jeder einzelnen wichtigen Stellungnahme realer Menschen kreuzen und verschlingen sich ja die Wertsphären. «11

Ein Zeitalter, das sich dank seines vermeintlichen Fortschritts von der kindlichen Vorstellung, es gebe »Götter ", unendlich weit entfernt wähnte, muß Weber zufolge feststellen, daß das Göttliche zwar sehr verschiedene Bezeichnungen tragen kann, daß es der Sache nach aber kein Jenseits bedeutet, das man beliebig bejahen oder verneinen kann, sondern dasjenige in der durchaus diesseitigen Wirklichkeit bedeutet, dem gegenüber jede »Beliebigkeit « ausgeschlossen ist, da es die jeweilige Form der Macht darstellt, die der Mensch in seinem Leben als die höchste anerkennt. Diese Einsicht eröffnet aber einen ganz neuen Blick auf die geistige Verfassung der vermeintlich vollends säkularisierten Moderne: »Die alten vielen Götter, entzaubert und

9 Die Suggestion lebt zum Teil von dem Wort »zeigen «, das im Alltagsgebrauch in den Bereich der wohlvertrauten visuellen Erfahrung gehört, wo der empirische Gegenstand, der sich anderen zeigen läßt, von vornherein als derselbe Gegenstand in den verschiedenen Akten des Zeigens verstanden wird.

10 Max Weber, Wissenschaft als Beruf (1917/1919), in: Ders., Gesamtausgabe, Bd. I/17, Tübingen 1992, 71-111, hier 99.

11 Max Weber, Der Sinn der »Wertfreiheit « der soziologischen und ökonomischen Wissenschaften (1914), in: Ders., Gesammelte Aufsätze zur Wissenschaftslehre, Tübingen ${ }^{4} 1973$, 489 540, hier 507. 
daher in Gestalt unpersönlicher Mächte, entsteigen ihren Gräbern, streben nach Gewalt über unser Leben und beginnen untereinander wieder ihren ewigen Kampf «. Und »über diesen Göttern und ihrem Kampf waltet das Schicksal, aber ganz gewiß keine 'Wissenschaft $\longleftarrow$. Es läßt sich nur verstehen, was das Göttliche für die eine und für die andere oder: in der einen und der anderen Ordnung ist. Damit ist aber die Sache für jede Erörterung in einem Hörsaal und durch einen Professor schlechterdings zu Ende, so wenig natürlich das darin steckende gewaltige Lebensproblem selbst damit zu Ende ist. Aber andere Mächte als die Katheder der Universität haben da das Wort. ${ }^{12}$

Wie der Schlaf der Vernunft Ungeheuer und Dämonen gebiert, so entfesselt die positivistische Verkürzung des Denkens den modernen Götterkampf der »Lebenswerte", dem die Gegenwart besonders hilflos ausgeliefert ist, weil sie der Versuchung nachgegeben hat, das anspruchsvolle Konzept der Vernunft durch das vermeintlich leichter und sicherer zu handhabende Konzept der positiven Wissenschaften zu ersetzen. Die fatalen Konsequenzen dieses Vorgangs hat Husserl in seinem Spätwerk zur Krise der europäischen Wissenschaften festgehalten: "Der positivistische Begriff der Wissenschaft in unserer Zeit ist also - historisch betrachtet - ein Restbegriff. Er hat alle die Fragen fallen gelassen, die man in die bald engeren, bald weiteren Begriffe von Metaphysik einbezogen hatte, darunter alle die unklar so genannten shöchsten und letzten Fragen`. Genau besehen haben sie $[. .$.$] ihre unabtrennbare Einheit darin, daß sie, sei es ausdrücklich,$ sei es in ihrem Sinn impliziert, die Probleme der Vernunft « enthalten. "Alle diese metaphysischen $<$ Fragen [...] übersteigen die Welt als Universum der bloßen Tatsachen. Sie übersteigen sie eben als Fragen, welche die Idee Vernunft im Sinne haben. Und sie alle beanspruchen eine höhere Dignität gegenüber den Tatsachenfragen, die auch in der Frageordnung unter ihnen liegen. Der Positivismus enthauptet sozusagen die Philosophie. «13

Die positivistische Reduktion der Vernunft, der verblendete Stolz der Moderne, glücklich in ein "nachmetaphysisches « Zeitalter eingetreten zu sein, die "Enthauptung " der Philosophie zugunsten der positiven Wissenschaften haben allesamt zur Folge, daß sich das menschliche Denken der Macht des Gegebenen und damit jeder gegebenen Macht ausliefert. Über die Mittel, wie das von der jeweils gerade anerkannten Lebensmacht Befohlene am effektivsten zu erreichen ist, soll gründlich nachgedacht werden; nicht aber darüber, ob es womöglich eine spezifische Macht der Vernunft gibt, vor der sich die einzelnen, untereinander konkurrierenden Lebensmächte noch einmal zu legitimieren hätten. Damit geraten aber die Lebensmächte in ein dämonisches, von keiner vernünftigen Sprache mehr zu erhel-

12 Weber, Wissenschaft (s.o., Anm. 10), $100 f$.

13 Edmund Husserl, Die Krisis der europäischen Wissenschaften und die transzendentale Phänomenologie. Eine Einleitung in die phänomenologische Philosophie, Gesammelte Werke, Bd. 6, Den Haag 1954, 6 f. 
lendes Dunkel, in dem ihre blinde Macht in genau dem Maße zunimmt, in dem das Licht abnimmt.

Soll angesichts dieser Situation die vermeintlich nüchterne Resignation Max Webers nicht das letzte Wort behalten, dann wird sich das Denken um eine Wiedergewinnung eines angemessenen Vernunftbegriffs bemühen müssen. Das durchaus nicht selbstverständliche Programm hierfür hat Adorno einmal in die pointierten Sätze gefaßt, es wäre "gegen Wittgenstein zu sagen, was nicht sich sagen läßt. Der einfache Widerspruch dieses Verlangens ist der von Philosophie selbst: er qualifiziert sie als Dialektik, ehe sie nur in ihre einzelnen Widersprüche sich verwickelt. Die Arbeit philosophischer Selbstreflexion besteht darin, jene Paradoxie auseinanderzulegen. ${ }^{14}$

$I V$.

Spätestens an dieser Stelle wird sich der Einwand nicht länger unterdrücken lassen, daß die letzten Überlegungen zwar einige möglicherweise interessante Aspekte des Verhältnisses von Vernunft und Religion im 20. Jahrhundert namhaft gemacht, daß sie aber nur sehr wenig mit Hegel zu tun haben. Dabei war doch am Beginn dieser Überlegungen in Aussicht gestellt worden, daß sie im Umweg über die Gegenwart einen Weg zu einem angemessenen, das heißt einem am zugrundeliegenden Sachproblem orientierten Verständnis Hegels eröffnen sollten. Mag also die pathologische Banalisierung der Vernunft zur positivistischen Wissenschaftlichkeit ein Signum des 20. Jahrhunderts darstellen, so würde dadurch die historische Distanz zu Hegel nur vergrößert, da seine Philosophie - so die allgemeine Überzeugung - doch gerade durch ein gleichsam »ungebrochenes "Vernunftvertrauen gekennzeichnet ist, das dem heutigen Denken im nostalgischen Rückblick vielleicht imponieren mag, das aber als Orientierungspunkt für ein zeitgemäßes Denken nicht ernsthaft in Frage kommen kann.

Es wird sich rasch zeigen, daß sich die Sache anders verhält. In seinem frühen Aufsatz »Glauben und Wissen « (1802) ruft Hegel, wie er es häufig und gern tut, zunächst ironisch ein allgemein verbreitetes Vorurteil des Zeitgeistes auf: »Über den alten Gegensatz der Vernunft und des Glaubens, von Philosophie und positiver Religion hat die Kultur die letzte Zeit [...] erhoben ", um dann sofort den prägnant formulierten Vorbehalt hinzuzusetzen: "Es ist aber die Frage, ob die Siegerin Vernunft nicht eben das Schicksal erfuhr, welches die siegende Stärke barbarischer Nationen gegen die unterliegende Schwäche gebildeter zu haben pflegt, der äußeren Herrschaft nach die Oberhand zu behalten, dem Geiste nach aber dem Über-

14 Theodor W. Adorno, Negative Dialektik. Gesammelte Schriften, Bd.6, Frankfurt a. M. 1973, 21. 
wundenen zu erliegen. « ${ }^{15}$ Allerdings wird - und dies ist Hegels eigentliche Pointe - nicht nur die Vernunft durch ihren zweideutigen Sieg korrumpiert, sondern ebenso die Religion: »Der glorreiche Sieg, welchen die aufklärende Vernunft über das, was sie nach dem geringen Maße ihres religiösen Begreifens als Glauben sich entgegengesetzt betrachtete, davongetragen hat, ist beim Lichte besehen kein anderer, als daß weder das Positive, mit dem sie sich zu kämpfen machte, Religion, noch daß sie, die gesiegt hat, Vernunft blieb.«16

Hegels Zeitdiagnose kann im Rückblick von heute wohl nicht anders als hellsichtig und zutreffend bezeichnet werden. Was sich das kritische Bewußtsein im 20. Jahrhundert gegen den übermächtigen Zeitgeist erst mühsam wieder erarbeiten muß, die Einsicht in die Dialektik der Aufklärung, daß der falsche Sieg der aufklärenden Vernunft zum genauen Gegenteil dessen geführt hat, was die Aufklärung ursprünglich verwirklichen wollte, - diese Einsicht liegt bereits, wie nun deutlich wird, dem Denken Hegels zu Grunde. Die Dialektik der Aufklärung wird von Hegel des Näheren als eine Dialektik der Säkularisierung begriffen, in deren Verlauf weder Vernunft noch Religion das bleiben, was sie ihrem eigenen Begriff nach sind. Dementsprechend breiten sich die Pathologien der Vernunft und die Pathologien des Glaubens immer stärker aus, weil sie sich in der mißlungenen Säkularisierung der Moderne gegenseitig verstärken. Denn eine Vernunft, die die »Lebensprobleme" der Menschen nicht mehr zu berühren vermag, ist eine pathologische und reduzierte Form der Vernunft. Dieser pathologischen Form der Vernunft antworten dann ebenso pathologische Kompensationsversuche, die das "ganz Andere" der Vernunft in einem pseudo-religiösen Jargon anpreisen - und womöglich für die eigentliche, »tiefere" Philosophie ausgeben.

Dadurch entsteht die nur auf den ersten Blick paradox anmutende Situation, daß eine Orientierung an Hegel für das heutige Denken nicht ein regressives "Zurück zu « darstellt, weil der vermeintliche »Fortschritt « der letzten Jahrhunderte sich in philosophischer Hinsicht als epochale Regression entpuppt, die hinter bereits erreichte Einsichten wieder in ältere, nunmehr freilich pathologisch verzerrte Denkmuster zurückfällt. Deshalb kann der zeitliche »Rückschritt" zu Hegel im kritischen Gegenzug hierzu den Ansatz zu einem »Fortschritt « in der Sache selbst bilden.

Nicht zufällig gehört Adorno, Mitautor der »Dialektik der Aufklärung ", im 20. Jahrhundert zu den ganz seltenen Ausnahmen derjenigen Denker, die das Verhältnis der Gegenwart zu Hegel in diesem Sinne verstanden haben. »In den abscheulichen Fragen ", was » an Hegel der Gegenwart etwas bedeute", klingt für Adorno eine verblendete "Anmaßung" mit.

15 Georg Wilhelm Friedrich Hegel, Glauben und Wissen, in: Ders., Gesammelte Werke, hg. v. Hartmut Buchner, Bd. 4, Hamburg 1968, 315-414, hier 315.

16 Ebd. 
Denn nicht »wird die umgekehrte Frage auch nur aufgeworfen, was die Gegenwart vor Hegel bedeutet; ob nicht etwa die Vernunft, zu der man seit seiner absoluten gekommen zu sein sich einbildet, in Wahrheit längst hinter jene zurückfiel und dem bloß Seienden sich anbequemte, dessen Last die Hegelsche Vernunft vermöge der im Seienden selbst waltenden in Bewegung setzen wollte. Alle Würdigungen fallen unter das Urteil aus der Vorrede der Phänomenologie des Geistes, das über jene ergeht, die nur darum über den Sachen sind, weil sie nicht in den Sachen sind. Sie verfehlen vorweg den Ernst und das Verpflichtende von Hegels Philosophie. «17

Das Sachproblem, von dem her sich Hegels Denken in einer angemessenen Weise erschließt, wird man daher nur in den Blick bekommen, wenn man nicht von vornherein seine Philosophie als wohlfeilen Steinbruch für vermeintlich »zeitgemäße« Doktrinen mißbraucht, sondern gerade jene zentralen Züge seiner Philosophie ernst nimmt, die zum heutigen Zeitgeist völlig quer stehen und den gegenwärtig approbierten Rationalitätsbegriff von Grund auf in Frage stellen. Damit kehrt der Gedankengang aber zur eingangs exponierten Verwandtschaft von Philosophie und Religion zurück. Denn für Hegel gilt: »Die Philosophie hat überhaupt Gott zum Gegenstand, und eigentlich zum einzigen Gegenstand. Philosophie ist keine Weltweisheit, wie man sie vormals so genannt hat; man hat sie so genannt im Gegensatz gegen den Glauben. Sie ist aber in der Tat nicht eine Weisheit der Welt, sondern eine Erkenntnis des Nichtweltlichen, keine Erkenntnis der äußerlichen Existenz, des empirischen Daseins und Lebens oder des formellen Universums, sondern sie ist Erkenntnis alles dessen, was ewig ist - dessen, was Gott ist und dessen, was seine Natur, indem sie sich manifestiert, entwickelt. «18

Daß diese Sätze quer zum heutigen Zeitgeist stehen, bedarf keiner näheren Begründung. Daraus zu folgern, sie seien deshalb falsch, wäre - das sollten die letzten Überlegungen deutlich machen - sicherlich voreilig. Daraus nun aber den umgekehrten Schluß zu ziehen, sie seien deshalb wahr, wäre töricht und unverantwortlich. Denn ihr Querstehen zum heute herrschenden Zeitgeist macht sie für das heutige Denken, also auch für ein Denken, das dem Zeitgeist kritisch gegenüberstehen mag, zunächst einmal schwer verständlich - und jedes rasche, unproblematische Verstehen wäre somit mit Sicherheit ein Mißverstehen. Daraus ergibt sich aber die konkrete Aufgabe, Hegels zentrales Lehrstück zum Verhältnis von Philosophie und Religion zunächst einmal möglichst nüchtern zu verstehen und in dieses Verständnis nach Möglichkeit verwandte Gedanken der klassischen deutschen Philosophie mit einzubeziehen.

\footnotetext{
17 Theodor W. Adorno, Drei Studien zu Hegel, in: Gesammelte Schriften, Bd. 5, Frankfurt a. M. 1970, 251.

18 Hegel, Religion (s.o., Anm. 1), 33 f.
} 
Das systematische Sachproblem, das hier zur näheren Bestimmung der epistemischen Verwandtschaft von Philosophie und Religion exponiert, nicht gelöst werden soll, betrifft eine grundlegende Schwierigkeit, die immer dann auftritt, wenn die Wirklichkeit als solche, das heißt das Ganze der Wirklichkeit thematisiert werden soll. Denn jedes einzelne Wirkliche, das wir empirisch erfahren und erkennen können, ist - wie es mit Hilfe einer räumlichen Metapher heißt - stets etwas in der Wirklichkeit (als ein Teil im Ganzen der Wirklichkeit). Dann ist aber im Umkehrschluß das Ganze der Wirklichkeit selbst nichts im angeführten Sinne Wirkliches, weil sie offenkundig nicht (wie ein Wirkliches) etwas in der Wirklichkeit sein kann (kein Teil in einem Ganzen). Wirkliches und Wirklichkeit sind somit nicht identisch - auch wenn es der Alltagssprache schwer fällt, diese epistemische wie ontologische Differenz angemessen auszudrücken.

Denn zur angemessenen Thematisierung des Ganzen der erfahrbaren Wirklichkeit bedarf es offenkundig anderer Begriffe, weil die »üblichen « Begriffe auf die Thematisierung von Einzelerfahrungen, das heißt von Teilbereichen und Ausschnitten der Wirklichkeit ausgerichtet sind. Oder anders gewendet: Bestimmte Begriffe mögen sehr gut geeignet sein, uns einen zuverlässigen Zugang zu einem bestimmten Teilbereich der Wirklichkeit zu eröffnen; gerade deshalb sind sie aber ungeeignet, das Ganze der Wirklichkeit als solches zu thematisieren. Freilich muß man sich hüten, aus dieser richtigen Einsicht den falschen Schluß zu ziehen, daß sich das Ganze deshalb aus prinzipiellen Gründen einer begrifflichen Artikulation entziehen muß (so daß es sich allenfalls im Schweigen "zeigen « würde). Denn die Forderung nach anderen Begriffen meint ja gerade nicht, auf Begriffe zu verzichten, sondern die Anstrengung, angemessenere Begriffe zu entwickeln und in eins damit die nur beschränkte Gültigkeit der üblichen Begriffe mit guten Gründen aufzuzeigen.

Dieser auf den ersten Blick nicht ganz einfache Gedanke hat seine klassische Formulierung bei Kant gefunden: »Jede einzelne Erfahrung ist nur ein Teil von der ganzen Sphäre ihres Gebietes, das absolute Ganze aller möglichen Erfahrung ist aber selbst keine Erfahrung und dennoch ein notwendiges Problem für die Vernunft, zu dessen bloßer Vorstellung sie ganz anderer Begriffe nötig hat, als jener reinen Verstandesbegriffe, deren Gebrauch nur immanent ist, d.i. auf Erfahrung geht. « ${ }^{19}$ Diese grundlegende Einsicht, daß das Ganze der Erfahrung selbst keine Erfahrung sein kann, hat für Kant freilich nicht die Konsequenz, daß das Ganze deshalb irgendwie »unbedeutender « oder »unwirklicher « wäre als die Einzelerfahrungen. Das genaue Gegenteil trifft zu: Das Ganze der erfahrbaren Wirklichkeit ist in dem präzisen Sinne »bedeutender " als jede Einzelerfahrung, weil es die

$19 \mathrm{KrV}$, AA IV 328. 
Bedingung der Möglichkeit jeder Teilerfahrung darstellt und sie infolgedessen erst zu dem macht, was sie ist. Deshalb ist das absolute Ganze aller möglichen Erfahrung ein notwendiges, nicht zu umgehendes Problem für die menschliche Vernunft.

Der grundlegende Ansatz für die Lösung des Problems besteht aber in der sorgfältigen Unterscheidung innerhalb der Rationalität zwischen Verstand und Vernunft. Deshalb sagt Kant mit großem Nachdruck: »Die Unterscheidung der Ideen, d.i. der reinen Vernunftbegriffe, von den Kategorien oder reinen Verstandesbegriffen, als Erkenntnissen von ganz verschiedener Art, Ursprung und Gebrauch, ist ein so wichtiges Stück [...], daß ohne eine solche Absonderung Metaphysik schlechterdings unmöglich oder höchstens ein regelloser, stümperhafter Versuch ist. «Daß Kant sein eigenes Werk ganz im Dienste der zu leistenden Grundunterscheidung versteht, stellt er unzweideutig klar, wenn er mit den Worten fortfährt: »Wenn Kritik der reinen Vernunft auch nur das allein geleistet hätte, diesen Unterschied zuerst vor Augen zu legen, so hätte sie dadurch schon mehr zur Aufklärung unseres Begriffs und der Leitung der Nachforschung im Felde der Metaphysik beigetragen, als alle fruchtlose Bemühungen, den transzendenten Aufgaben der reinen Vernunft ein Gnüge zu tun, die man von je her unternommen hat, ohne jemals zu wähnen, daß man sich in einem ganz andern Felde befände als dem des Verstandes und daher Verstandes- und Vernunftbegriffe, gleich als ob sie von einerlei Art wären, in einem Striche hernannte. «" ${ }^{20}$ Oder anders ausgedrückt: Philosophie als Metaphysik ist die Anstrengung der Vernunft, das mit eigenen Begriffen zu sagen, was sich mit Verstandesbegriffen nicht sagen läßt.

Die genuine Aufgabe der Philosophie, eine andere Begrifflichkeit als die des auf Einzelerfahrungen fixierten Alltagsbewußtseins (das heißt für Kant: des Verstandes) zu entwickeln, markiert damit das zentrale Sachproblem eines Denkens, das sich weder in die Grenzen des Verstandespositivismus einsperren und neutralisieren lassen will, noch den bequemen "Sprung " in ein mystisches Schweigen für sinnvoll hält, weil er die Herrschaft des Verstandes auf dem Gebiet der begründeten Rede unangefochten läßt. Kant versucht dieses Problem durch den Nachweis zu lösen, daß das Verstandesdenken in seiner beschränkten Fixiertheit auf das "positiv « gegebene Einzelne und empirisch Erfahrbare überhaupt nur möglich ist, weil es unbewußt das unerfahrbare Ganze der Erfahrung als unthematischen Horizont des Einzelnen immer schon in Anspruch genommen hat.

Die innere Dynamik des Verstandesdenkens, die rastlos über jede bestimmte Einzelerkenntnis zur nächsten hinausdrängt, verrät deshalb indirekt diese immanente Bezogenheit des Verstandes auf die Vernunft. Dabei ist für den hier verfolgten Gedankengang von besonderer Bedeutung, daß Kant, der den internen Zusammenhang von Verstand und Vernunft in der Kritik

$20 \mathrm{KrV}$, AA IV $328 f$. 
der reinen Vernunft vermittels einer eigens hierfür entwickelten transzendentalen Terminologie analysiert, nicht zögert, ihn auch in religiösen Begriffen auszudrücken: "Daher sehen wir bei allen Völkern durch ihre blindeste Vielgötterei doch einige Funken des Monotheismus durchschimmern, wozu nicht Nachdenken und tiefe Spekulation, sondern nur ein nach und nach verständlich gewordener natürlicher Gang des gemeinen Verstandes geführt hat. « ${ }^{21}$ Die immanente kritische Opposition der Vernunft gegenüber dem Partikularismus des Verstandes hat demnach ihre religiöse Entsprechung in der immanenten kritischen Opposition des Monotheismus gegenüber dem Polytheismus. ${ }^{22}$

Diese philosophisch-religiöse »Doppelcodierung « seiner grundlegenden Einsichten ist bei Kant durchaus kein Einzelfall. So faßt er den großen Gedankengang seiner Vernunftphilosophie ${ }^{23}$ von der Kritik der reinen Vernunft über die Kritik der praktischen Vernunft zur Kritik der Urteilskraft rückblickend in der letzten Kritik ebenfalls in religiösen Begriffen noch einmal zusammen: »Mit diesem allem soll hier nur so viel gesagt werden: daß die Furcht zwar zuerst Götter (Dämonen), aber die Vernunft vermittelst ihrer moralischen Principien zuerst den Begriff von Gott habe hervorbringen können. « ${ }^{24}$ Vermittels ihrer moralischen Prinzipien vermag die Vernunft nämlich die menschlichen Entscheidungen am Maßstab ihrer strikten Verallgemeinerbarkeit, das heißt am Maßstab des Ganzen auszurichten, so daß der Partikularismus der miteinander konkurrierenden Werte überwunden wird, der notwendig im Rahmen des Verstandesdenkens das letzte Wort haben muß, weil der Verstand auch darin beschränkt ist, daß er nur die Mittel für gegebene Zwecke bestimmen, aus sich heraus aber keine Zwecke setzen kann, sondern dies den vielen sinnlichen Affekten überlassen muß, in deren Konkurrenz die Furcht häufig den Sieg davonträgt.

Kants Moralphilosophie der reinen praktischen Vernunft hat sich von Beginn an dem Einwand ausgesetzt gesehen, daß reine Vernunft für sich genommen nicht in der Lage ist, das menschliche Handeln zu motivieren, es sei denn, sie nähme am Ende doch versteckt sinnliche Antriebe zu Hilfe (womit sie freilich auf die Ebene des Verstandes regredieren würde). Kant hat sich mehrfach gegen diesen Einwand zur Wehr gesetzt; am nachdrücklichsten vielleicht dort, wo er erneut zu religiösen Begriffen greift: »Vielleicht gibt es keine erhabenere Stelle im Gesetzbuche der Juden, als das Gebot: Du sollst

${ }^{21} \mathrm{KrV}, \mathrm{B} 618$.

22 Die immanente kritische Bezogenheit des Monotheismus auf den Polytheismus hat in der klassischen deutschen Philosophie vor allem Schelling hervorgehoben, wenn er sagt: Ist »der Polytheismus eine Unmöglichkeit, so ist Monotheismus als besonderer Begriff nicht weniger eine Unmöglichkeit. Beide Begriffe stehen und fallen miteinander « (SW XII 15); vgl. hierzu vom Verf.: Geschichtliche Vernunft. Die Weiterführung der Kantischen Vernunftkritik in der Spätphilosophie Schellings, Frankfurt a. M. 1996.

23 Vgl. hierzu vom Verf.: Das Interesse der Vernunft. Kants ursprüngliche Einsicht und ihre Entfaltung in den transzendentalphilosophischen Hauptwerken, Hamburg 2003.

$24 \mathrm{KrV}, \mathrm{AA} \mathrm{V} 447$. 
dir kein Bildnis machen, noch irgend ein Gleichnis, weder dessen, was im Himmel, noch auf der Erden, noch unter der Erden ist u.s.w. Dieses Gebot allein kann den Enthusiasmus erklären, den das jüdische Volk in seiner gesitteten Epoche für seine Religion fühlte, wenn es sich mit andern Völkern verglich, oder denjenigen Stolz, den der Mohammedanismus einflößt. Eben dasselbe gilt auch von der Vorstellung des moralischen Gesetzes und der Anlage zur Moralität in uns. Es ist eine ganz irrige Besorgnis, daß, wenn man sie alles dessen beraubt, was sie den Sinnen empfehlen kann, sie alsdann keine andere als kalte, leblose Billigung und keine bewegende Kraft oder Rührung bei sich führen würde. Es ist gerade umgekehrt. «"

Es muß im begrenzten Rahmen der vorliegenden Überlegungen dahingestellt bleiben, wie weit der hier abermals von Kant kühn gespannte Bogen zwischen Vernunftphilosophie und Monotheismus wirklich trägt. Für den Zweck des hier verfolgten Gedankengangs genügt es festzuhalten, daß bereits Kant mit großem Nachdruck der Überzeugung ist, die auch Hegel vertritt, daß die Philosophie sich selbst expliziert, indem sie die Religion expliziert, und daß sie, indem sie sich expliziert, die Religion expliziert.

VI.

Die epistemische Verwandtschaft von Philosophie und Religion liegt nach Hegels Selbstverständnis seiner gesamten Philosophie zugrunde, wird aber insbesondere in der Philosophie der Religion eigens zum Thema. Hegels religionsphilosophische Vorlesungen beginnen daher mit der allgemeinen »Erinnerung «, welchen »Gegenstand wir in der Religionsphilosophie vor uns haben. Es ist der Gegenstand der Religion selbst; er ist der höchste, absolute Gegenstand. ${ }^{26}$ Die in diesem Satz leicht zu übersehende Nuance ist entscheidend: Hegels Religionsphilosophie hat durchaus nicht die Religion zum Gegenstand, sondern den Gegenstand der Religion. Hierin unterscheidet sich die Philosophie der Religion von jeder einzelwissenschaftlichen (historischen, soziologischen, psychologischen) Beschäftigung mit der Religion, daß sie die Religion nicht als positiv gegebenen Gegenstand auffaßt, sondern sie in ihrem eigenen Wahrheitsanspruch ernst nimmt, eine geistige Auseinandersetzung mit einem von der Religion selbst unterschiedenen » Gegenstand « zu sein.

Dieser Gegenstand der Religion ist aber kein bestimmter Gegenstand neben anderen (weshalb der Begriff "Gegenstand « hier leicht mißverstanden werden kann), sondern das »Höchste « oder das »Absolute «. Der Gegenstand wird dergestalt zunächst nicht inhaltlich, sondern formal bestimmt als das, worüber hinaus nichts Größeres zu denken ist, also als das Ganze,

$25 \mathrm{KrV}, \mathrm{AA}$ V 274.

26 Hegel, Religion (s.o., Anm. 1), 31. 
durch das alles Partikulare bedingt, das aber selbst unbedingt ist. Die nähere inhaltliche Bestimmung dieser Vernunftidee ist nicht ohne Schwierigkeiten, so daß es nicht überraschen kann, daß Hegel gleich eine ganze Reihe von Begriffen aufbietet, die als begriffliche Variationen über ein einziges Thema zu verstehen sind. Der Gegenstand der Religion ist die Region der »Wahrheit «; ein Gegenstand, von dem sich weiter sagen läßt, »daß er um seiner selbst willen « ist: »Er ist schlechthin an und für sich das Unbedingte, Freie, Grenzenlose, das nur sich selber Zweck und Endzweck ist. « ${ }^{27}$ Die verschiedenen Philosophien und die verschiedenen Religionen beziehen sich alle auf diesen gemeinsamen Gegenstand, das macht ihre Verwandtschaft und Vergleichbarkeit aus, doch beziehen sie sich in je unterschiedlicher Weise und zudem in einer eher angemessenen oder unangemessenen Weise auf ihn, das macht ihren Unterschied und ihre Rangordnung aus.

Darüber hinaus gibt es aber noch einen grundlegenderen Unterschied zwischen der Philosophie einerseits und der Religion andererseits, der Hegels Lehre von der systematischen Verwandtschaft von Philosophie und Religion am Ende ihr konkretes, in sich differenziertes Profil verleiht. Ein solchermaßen grundlegender Unterschied wird bei Hegel stets vor dem Hintergrund einer Gemeinsamkeit angegeben. Im Falle von Philosophie und Religion besteht die Gemeinsamkeit nicht nur, wie bereits ausführlich dargelegt, in einem gemeinsamen Gegenstand, sondern zusätzlich darin, daß sich Philosophie und Religion nicht beim positiv Gegebenen der Alltagserfahrung beruhigen, sondern in jeweils eigener Weise nach der nicht-empirischen "Bedeutung " des empirisch Gegebenen fragen. Die "Verschiedenheit" von Philosophie und Religion ist deshalb für Hegel nur angemessen »bemerklich zu machen", wenn "wir zunächst betrachten, was >Bedeuten selbst bedeutet «. Denn indem »wir fragen, was bedeutet dies oder jenes, so wird nach zweierlei gefragt, und zwar nach dem Entgegengesetzten. ${ }^{28}$

Die eine Bedeutung von Bedeutung ist leitend, wenn wir einen strittigen Ausdruck oder ein Kunstwerk vor uns haben und nun nach der eigentlichen »Bedeutung « fragen. Die Frage geht hier, so Hegel, auf »den Sinn, den Zweck, allgemeinen Gedanken jenes Ausdrucks, Kunstwerks, das Innere." Diese »innere " Bedeutung ist dabei stets eine gedankliche Bestimmung: »Wenn wir so fragen, was ist Gott, was bedeutet der Ausdruck >Gott<, so wollen wir den Gedanken, der soll uns angegeben werden, die Vorstellung haben wir wohl. Sonach hat es die Bedeutung, daß der Begriff angegeben werden soll, und so ist allerdings das, was wir in der Philosophie das Absolute oder die Idee nennen, die Bedeutung; es ist das Absolute, die Idee, die begriffene Natur Gottes, die in Gedanken gefaßte Natur Gottes, oder das logische Wesen desselben, was wir wissen wollen. $\ll^{29}$

27 Ebd.

28 AaO., 34.

29 Ebd. 
Die andere Bedeutung von Bedeutung besitzt einen Sinn, »der das Entgegengesetzte verlangt. Nämlich wenn wir von reinen Gedankenbestimmungen anfangen, und nicht von der Vorstellung, so kann es der Fall sein, daß der Geist sich in dieser Gedankenbestimmung nicht befriedigt findet, nicht darin zu Hause ist, und fragt, was diese reine Gedankenbestimmung zu bedeuten habe. So findet sich z.B. die Bestimmung von Einheit des Subjektiven und Objektiven, von Einheit des Realen und Ideellen; man kann jedes für sich verstehen - wissen, was Einheit, Objektives, Subjektives usf. ist, und doch kann man sehr wohl sagen, man verstehe diese Bestimmung nicht. Wenn wir in einem solchen Fall fragen, so ist >Bedeutung das Entgegengesetzte von vorher. Hier wird eine Anschauung, Vorstellung der Gedankenbestimmung gefordert. « $^{30}$

Der Satz »Gott ist der unbedingte Selbstzweck « ist ein genuin philosophischer Satz, weil er eine gegebene Vorstellung gedanklich durchdringt, auf den Begriff bringt und dergestalt die ebenso vage wie kontroverse Bedeutung des Ausdrucks »Gott « näher bestimmt. Hingegen ist der Satz »Die absolute Idee ist Gott " ein genuin religiöser Satz, da er eine reine Gedankenbestimmung paradox veranschaulicht, das heißt das, was sich nicht veranschaulichen läßt, gleichwohl veranschaulicht; das, wovon sich der Mensch kein Bild machen soll, gleichwohl in eine reale Vorstellung überführt und dergestalt die ebenso abstrakte wie kontroverse Bedeutung des Ausdrucks »absolute Idee« näher bestimmt.

Für Hegel ist freilich die wahre und gültige Bedeutung, auf die es am Ende ankommt, erst die dialektische Einheit der beiden soeben unterschiedenen Bedeutungsdimensionen. Denn wenn »wir so von der Vorstellung Gottes anfangen und nach der Bedeutung fragen, so ist einerseits die Idee von Gott verlangt, das Absolute, das im Begriff erfaßte Wesen, und diese Bedeutung ist gemeinsam mit der logischen Idee; aber Gott ist dies, nicht nur an sich zu sein, er ist ebenso wesentlich für sich. Er ist Geist, und nicht der endliche, sondern der absolute Geist, und dies, daß er Geist ist, ist das, daß er nicht nur das im Gedanken sich haltende Wesen ist, sondern auch das Erscheinende, das sich Offenbarung, das sich Gegenständlichkeit Gebende ist. " ${ }^{31}$ Die Philosophie der Religion geht also in diesem entscheidenden Punkt über die Wissenschaft der Logik hinaus, in der die reinen Gedankenbestimmungen untersucht werden: »In der Religionsphilosophie haben wir so das Absolute zum Gegenstand, aber nicht bloß in der Form des Gedankens, sondern auch in der Form seiner Manifestation. - Die allgemeine Idee ist also zu fassen in ihrer schlechthin konkreten Bedeutung, worin die Bestimmung liegt, zu erscheinen, sich zu offenbaren. Diese Seite des Daseins ist aber selbst wieder in der Philosophie zu denken und mit dem Gedanken zu fassen. « ${ }^{32}$

30 AaO., 34f.

$31 \mathrm{AaO}, 35$.

32 AaO., 36. 
Die Wirklichkeit philosophisch, das heißt vernünftig zu begreifen, erfordert demnach bei Hegel eine Doppelbewegung. Zum einen ist deutlich zu machen, daß das Wirkliche vernünftig ist. Dies erfordert eine gedankliche Durchdringung der anschaulich und in Vorstellungen gegebenen Wirklichkeit. Zum anderen ist zugleich deutlich zu machen, daß das Vernünftige wirklich ist, daß es sich immer schon manifestiert und verwirklicht hat. Ersteres ist die Aufgabe der Philosophie; letzteres ist das Thema der Religion. Daß beide zunächst unterschiedenen Zugangsweisen zur Wahrheit am Ende eine in sich differenzierte Einheit bilden - dies zu explizieren, ist wiederum Aufgabe der Philosophie. ${ }^{33}$ Deshalb ist für Hegel eine Philosophie der Religion möglich, ja notwendig, nicht aber umgekehrt eine Religion der Philosophie.

\section{VII.}

Am Ende gibt Hegel noch eine Gegenprobe auf sein zentrales Lehrstück, indem er die pathologischen Konsequenzen aufzeigt, die sich gemäß einer inneren Logik ergeben müssen, sobald die von ihm behauptete Wahrheit verfehlt wird. Im Falle der systematischen Verwandtschaft von Philosophie und Religion führt dies bei Hegel zur kritischen Betrachtung einer Theologie, die nicht länger den Gegenstand der Religion zum Thema hat, sondern nur noch die Religion selbst: " $\mathrm{Zu}$ bemerken ist, daß es eine Theologie gibt, die sich in Ansehung der Religion nur historisch verhalten will, die auch eine Fülle von Erkenntnissen hat, die jedoch nur historischer Art sind. Dies Erkennen geht uns nichts an; wäre das Erkennen der Religion nur historisch, so müßten wir solche Theologen mit Kontorbedienten eines Handelshauses vergleichen, die nur über fremden Reichtum, der ihnen durch die Hände geht, von dem sie aber nichts behalten, Buch und Rechnung führen, die nur für andere handeln, ohne eigenes Vermögen zu bekommen; sie erhalten zwar Salär; ihr Verdienst ist aber nur, zu registrieren, was das Vermögen anderer ist. In der Philosophie und Religion ist es aber wesentlich darum zu tun, daß der eigene Geist sich einen Besitz und Inhalt erkennt, sich der Erkenntnis für würdig hält, nicht demütig sich draußen hält. «34

Diese Kritik voll beißender Ironie muß fairerweise durch den Fall einer Philosophie ergänzt werden, die sich selbst nur noch historisch versteht, weil sie nur noch die positiv gegebenen Texte Platons oder Kants zum Gegenstand hat, aber nicht mehr das, was Platon oder Kant selbst zum Gegenstand ihres Denkens machten. Beides zusammengenommen, die Histori-

\footnotetext{
33 Vgl. Robert Spaemann, Die Frage nach der Bedeutung des Wortes `Gott‘, in: Ders., Einsprüche, Einsiedeln 1977, 13-35.

34 Hegel, Religion (s.o., Anm. 1), 44.
} 
sierung der Theologie und der Philosophie, macht aber den Kern des fatalen Siegeszuges der sogenannten "Geisteswissenschaften " ab der zweiten Hälfte des 19. Jahrhunderts aus. Ihn hat zuerst Nietzsche als epochale Pathologie gebrandmarkt, deren Leitsymptom ein »überschwemmendes, betäubendes und gewaltsames Historisieren « ist. Insbesondere der Unterricht wird hierdurch verdorben: »Der junge Mensch wird durch alle Jahrtausende gepeitscht «, und »die Masse des Einströmenden ist so groß, das Befremdende « dringt so übermächtig »auf die jugendliche Seele ein, daß sie sich nur mit einem vorsätzlichen Stumpfsinn zu retten weiß. $\ll^{35}$ Der historische Sinn, auf den die Moderne so stolz ist, wird dergestalt zur Grundlage einer immer weiter um sich greifenden Geist- und Vernunftfeindschaft.

Nietzsche spricht in diesem Zusammenhang von einer pathologischen Musealisierung des Geistes: Wie »der junge Mensch durch die Geschichte läuft, so laufen wir Modernen durch die Kunstkammern, so hören wir Konzerte. Man fühlt wohl, das klingt anders als jenes, das wirkt anders als jenes: dies Gefühl der Befremdung immer mehr zu verlieren, über nichts mehr übermäßig zu erstaunen, endlich alles sich gefallen zu lassen - das nennt man dann wohl den historischen Sinn, die historische Bildung. «36 Heidegger nimmt Nietzsches Diagnose auf und entwickelt sie weiter, wenn er schreibt: "Die Lage Europas ist um so verhängnisvoller, als die Entmachtung des Geistes aus ihm selbst herkommt und - wenn auch durch Früheres vorbereitet - sich endgültig aus seiner eigenen geistigen Lage in der ersten Hälfte des 19. Jahrhunderts bestimmt. Bei uns geschah um jene Zeit das, was man gern und kurz als den 'Zusammenbruch des deutschen Idealismus bezeichnet. Diese Formel ist gleichsam ein Schutzschild, hinter dem sich die schon anbrechende Geistlosigkeit, die Auflösung der geistigen Mächte, die Abwehr alles ursprünglichen Fragens nach Gründen und die Bindung an solche verstecken und decken. Denn nicht der deutsche Idealismus brach zusammen, sondern das Zeitalter war nicht mehr stark genug, um der Größe, Weite und Ursprünglichkeit jener geistigen Welt gewachsen zu bleiben. $\ll^{37}$

Für ein Denken, das nicht einfach im Zeitgeist der Gegenwart aufgehen will, erwächst hieraus die nicht triviale Aufgabe, an die Konzepte der klassischen deutschen Philosophie in einer Weise zu erinnern, daß diese Lehrstücke gerade aus den »Kunstkammern « der Philosophiegeschichte befreit werden. Bei solch einem Unternehmen kommt aber der Erinnerung an die systematische Verwandtschaft von Philosophie und Religion eine zentrale Bedeutung zu. Die Philosophie nicht bloß historisch, sondern auf die in ihr verhandelte Sache hin zu verstehen, bedeutet nämlich, sie auf den Gegenstand hin zu verstehen, den sie mit der Religion gemeinsam hat.

\footnotetext{
35 Friedrich Nietzsche, Vom Nutzen und Nachteil der Historie für das Leben, Kritische Gesamtausgabe, Bd. III/1, Berlin 1972, 239-330, $295 \mathrm{f}$.

36 AaO., 295.

37 Martin Heidegger, Einführung in die Metaphysik, Tübingen ${ }^{5} 1987,34 f$.
} 


\section{ZUSAMMENFASSUNG}

Das verdrängte Sachproblem, das der Aufsatz in Erinnerung rufen will, ist die systematische und epistemische Verwandtschaft von Philosophie und Religion. Verdrängt wurde dieses Sachproblem im Zuge der positivistischen und historistischen Verkürzung des Denkens: Wie der Schlaf der Vernunft Ungeheuer und Dämonen gebiert, so entfesselt die Verkürzung des Denkens den modernen »Polytheismus der Lebenswerte (Max Weber), dem die Gegenwart besonders hilflos ausgeliefert ist, weil sie der Versuchung nachgegeben hat, das anspruchsvolle Konzept der Vernunft durch das vermeintlich leichter und sicherer zu handhabende Konzept der positiven Wissenschaften zu ersetzen. Eine solche Dialektik der Aufklärung läßt sich mit Kant und Hegel des Näheren als eine Dialektik der Säkularisierung begreifen, in deren Verlauf weder Vernunft noch Religion das bleiben, was sie ihrem eigenen Begriff nach sind. Dementsprechend breiten sich die Pathologien der Vernunft und die Pathologien des Glaubens immer stärker aus, weil sie sich in der mißlungenen Säkularisierung der Moderne gegenseitig verstärken. Denn eine Vernunft, die die "Lebensprobleme« der Menschen nicht mehr zu berühren vermag, ist eine pathologische und reduzierte Form der Vernunft. Dieser pathologischen Form der Vernunft antworten dann ebenso pathologische Kompensationsversuche, die das "ganz Andere « der Vernunft in einem pseudo-religiösen Jargon anpreisen - und womöglich für die eigentliche, »tiefere« Philosophie ausgeben. Im kritischen Gegenzug hierzu will der Aufsatz an die Einsicht erinnern, daß die Philosophie, als Wissenschaft der Wahrheit, nur sich selbst expliziert, indem sie den Wahrheitsgehalt der (monotheistischen) Religion expliziert, und indem sie umgekehrt das Wesen der (philosophischen) Wahrheit expliziert, expliziert sie die Religion.

\section{SUMMARY}

This essay calls to mind the suppressed problem of the systematic and epistemic relationship between philosophy and religion. This substantive issue has been suppressed by reductionist views of thinking which followed in the wake of positivism and historicism. Just as the sleep of reason brings forth monsters and demons, so the reductionist tendencies of thinking unleash the "polytheism of the values of life" (Max Weber). Our present age is helplessly at the mercy of these tendencies because it has given in to the temptation of replacing the ambitious concept of reason with the concept of the positive sciences which are supposedly easier to handle and can more successfully fulfil the promise of assured results. Such a dialectic of enlightenment can be conceived as a dialectic of secularisation, in the course of which neither reason nor religion remain what they are according to their own genuine conception. Consequently, the pathologies of reason and the pathologies of faith spread ever more rapidly, because they enhance one another in the context of the failed secularisation of modernity. A form of reason that is no longer in touch with the problems of human life is a pathological and reductionist form of reason. Strategies of compensation, however, which celebrate the "totally other" of reason in a pseudo-religious jargon, possibly even presenting it as the real, the "deeper" form of philosophy, are no less pathological. In contrast to such tendencies, this essay is intended to serve as a reminder of the insight that philosophy as the science of truth only explicates itself by explicating (monotheistic) religion, and only explicates religion by explicating the character of (philosophical) truth. 